\title{
Artificially Induced Phase Scintillation Observed From GLONASS Signals
}

This paper was downloaded from TechRxiv (https://www.techrxiv.org).

LICENSE

CC BY 4.0

SUBMISSION DATE / POSTED DATE

$16-11-2021 / 29-11-2021$

\section{CITATION}

Darya, Abdollah; Shaikh, Muhammad; Fernini, Ilias; AINaimiy, Hamid (2021): Artificially Induced Phase Scintillation Observed From GLONASS Signals. TechRxiv. Preprint.

https://doi.org/10.36227/techrxiv.17021936.v1

DOI

10.36227/techrxiv.17021936.v1 


\title{
Artificially Induced Phase Scintillation Observed From GLONASS Signals
}

\author{
Abdollah Masoud Darya, Member, IEEE, Muhammad Mubasshir Shaikh, Ilias Fernini, and Hamid AlNaimiy
}

\begin{abstract}
The phase scintillation index is a commonly used metric in the remote sensing of ionospheric irregularities. In this work, we analyze the phase scintillation index observed from the GPS, GLONASS, Galileo, and BeiDou satellite constellations, for a continuous period of three years. Our analysis reveals an elevated level of $L 1$ phase scintillation observed from most GLONASS satellites, and non of the other GNSS constellations during the same period. This is of particular interest as the abnormality was observed during a solar minimum period, and from satellites which were labeled as healthy. Furthermore, the observations made were verified with data from three other receivers in different regions. This study was conducted to highlight these artificially induced phase scintillations from GLONASS satellites so that future studies can take them into considerations, especially during periods of heightened geomagnetic activity. Additionally, these artificially induced phase scintillations may result in loss of phase lock, as well as reduced positioning accuracy, which may have serious effects on the reliability and integrity of the GLONASS positioning service.
\end{abstract}

\section{INTRODUCTION}

$\mathbf{T}$ HE presence of ionospheric irregularities in the path of satellite-to-ground signals induces amplitude and phase fluctuations. These fluctuations severely limit the performance of global navigation satellite systems (GNSS) and may cause loss of receiver lock [1]. Continuous monitoring of phase scintillation of GNSS signals is used to remotely sense ionospheric irregularities [2]. Phase scintillation is typically monitored through the Sigma-Phi $\left(\sigma_{\phi}\right)$ index by ionospheric scintillation monitoring GNSS receivers [3], [4]. Since $\sigma_{\phi}$ is a commonly used metric to remotely sense ionospheric irregularities in the signals path, any abnormally elevated values of $\sigma_{\phi}$ must be studied and highlighted.

Traditionally, researchers have relied exclusively on the GPS constellation for $\sigma_{\phi}$ measurements [5]-[9]. However, with the availability of the GLONASS, Galileo, and BeiDou constellations, more data is now available, thus providing better coverage of the ionospheric scintillations. Furthermore, the BeiDou constellation combines satellites at geostationary orbits (GEO) and inclined GEO (IGSO), in addition to medium-earth-orbit (MEO) satellites [10]. This allows for studying the ionosphere from different viewpoints.

This work presents the findings of our three year study into the artificially induced phase scintillation observed on L1 signals from GLONASS satellites. During the same period, This artificially induced phase scintillation was not observed on GPS, Galileo, and BeiDou L1 signals. Observations from

The authors are with the Sharjah Academy for Astronomy, Space Sciences and Technology, University of Sharjah, UAE (e-mail: adarya@sharjah.ac.ae; mshaikh@sharjah.ac.ae; ifernini@sharjah.ac.ae; alnaimiy@sharjah.ac.ae).
TABLE I

INFORMATION ABOUT THE STATIONS UTILIZED IN THIS WORK.

\begin{tabular}{|c|c|c|c|}
\hline Station & Data Availability & Latitude & Longitude \\
\hline$m 02 c$ & 01-Jan-2019 to 05-Feb-2019 & 18.35 & -66.75 \\
\hline$m 07 c$ & 01-Jan-2019 to 31-Mar-2019 & 64.78 & -141.16 \\
\hline$m 12 c$ & 16-Jan-2019 to 31-Mar-2019 & 51.15 & -107.26 \\
\hline SHJ1 & 09-Oct-2018 to 09-Oct-2021 & 25.28 & 55.46 \\
\hline
\end{tabular}

different stations at different geographical regions were used to confirm the presence of this abnormality. Furthermore, this study was conducted during a solar minimum period to ensure that $\sigma_{\phi}$ observations were not affected due to enhancements in geomagnetic activity.

Although [1] utilized L1 GPS and GLONASS phase scintillation data in their study, they did not report any abnormally elevated phase scintillation values. That may have been because they aimed to study the correlation between phase scintillation and the receiver tracking performance, and as such they only utilized six days of observation data from a single GNSS station. Since then, as far as the authors are aware, there have been no works that reported abnormally elevated values of phase scintillation from the GLONASS constellation. There also have not been any works that reported any long-term abnormality in the operation of the GLONASS constellation.

The outline of this work is as follows. First, in section [II, information about the utilized data is presented. Then, the observations are presented in section III. Finally, the conclusions are given in section IV.

\section{DATA}

In this work, we have used three-year L1 $\sigma_{\phi}$ data obtained from a multi-frequency, multi-constellation GNSS receiver (PolaRx5S) with scintillation measurement capabilities, located at the Sharjah Academy for Astronomy, Space Sciences, and Technology (SHJ1). Data from three other stations, $\mathrm{mO2c}$, $m 07 c$, and $m 12 c$, located in different regions was also considered in this work for comparison (see Table I). These three stations are part of the Monitors for the Alaskan and Canadian Auroral Weather in Space (MACAWS) network. Their data is freely available at http://cedar.openmadrigal.org. The L1 $\sigma_{\phi}$ considered in this work is defined as the standard deviation, in radians, of the $50 \mathrm{~Hz}$ detrended carrier phase averaged over a 1-minute interval $\left(\phi_{60}\right)$. The phase detrending was done by filtering the raw $50 \mathrm{~Hz}$ carrier phase measurements by a 6th order Butterworth high-pass filter with a cutoff frequency of $0.1 \mathrm{~Hz}$ [3]. Additionally, an elevation cutoff of $15^{\circ}$ was used in this work. We also used GLONASS satellite health data 


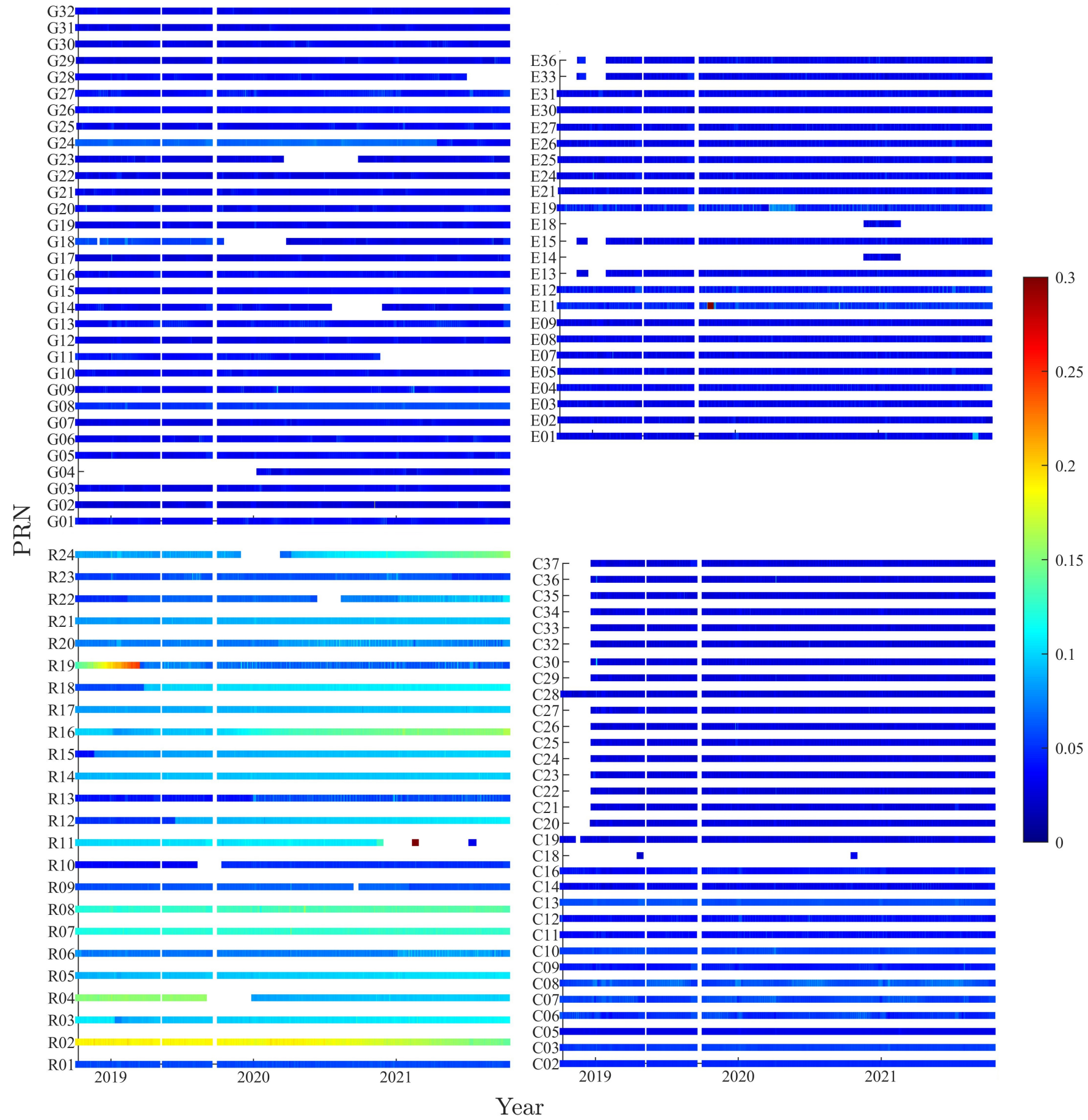

Fig. 1. The 6-hour averaged L1 $\sigma_{\phi}$ observed on GPS (G01-G32), GLONASS (R01-R24), Galileo (E01-E36), and BeiDou (C02-C37) satellites at the SHJ1 station over a period of three years. The color bar represents $\sigma_{\phi}$ ranging from 0 to $\geq 0.3$. The y-axis represents the RINEX PRN/SN and the x-axis represents the time in years. 


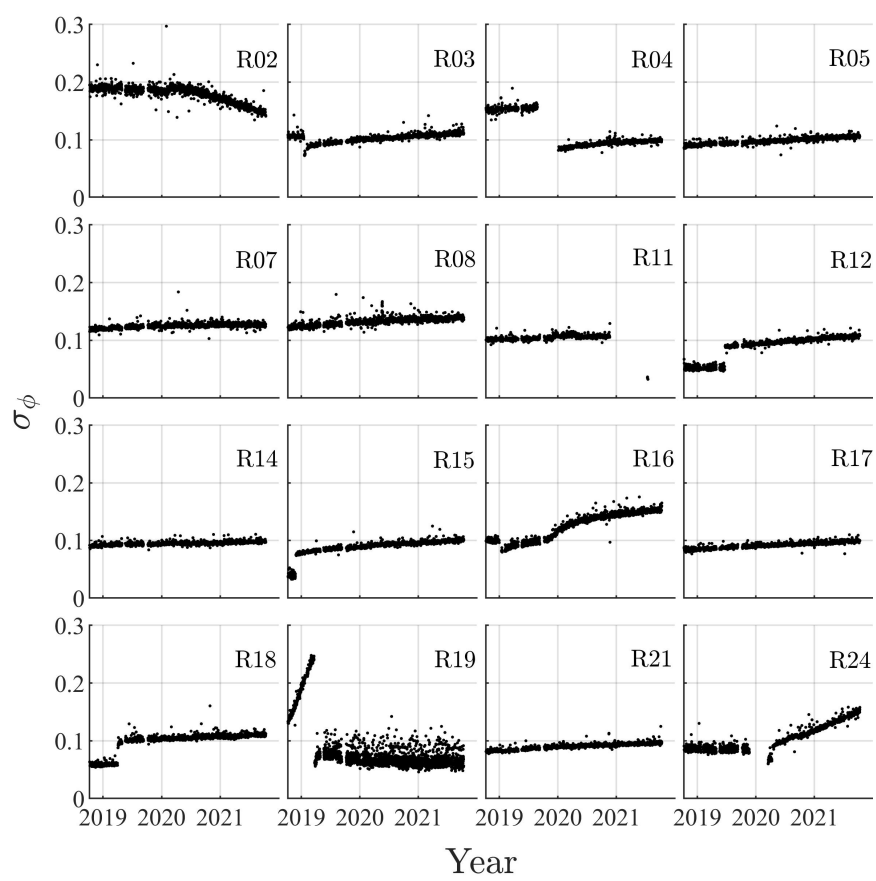

Fig. 2. The 6-hour averaged L1 $\sigma_{\phi}$ trends of the 16 most-affected GLONASS satellites observed at the SHJI station over a period of three years. The y-axis represents $\sigma_{\phi}$, and the $\mathrm{x}$-axis represents the time in years.

taken from GLONASS navigation ephemeris files which are available at the international GNSS service (IGS) broadcast service (https://cddis.nasa.gov).

\section{FINDINGS AND DISCUSSION}

In Fig. 1] we present $\sigma_{\phi}$ observations for the four major GNSS constellations. The different GPS, Galileo, and BeiDou satellites will be referred to by their Receiver INdependent EXchange format (RINEX) Pseudo-Random Noise (PRN) codes, while the GLONASS satellites will be referred to by their Slot Number (SN).

Fig. 1 shows the 6-hour averaged L1 $\sigma_{\phi}$ trends observed for GPS (G01-G32), GLONASS (R01-R24), Galileo (E01-E36), and BeiDou (C02-C37) satellites over a period of three years, from 09-Oct-2018 to 09-Oct-2021. The 6-hour average trends have been used in this case to identify the long-term variation of the artificially induced $\sigma_{\phi}$ and to remove any naturally caused temporal variation.

As can be seen, all GPS, Galileo, and BeiDou satellites maintain consistent levels of $\sigma_{\phi}<0.1$. The only exceptions are G24 and E19 that experienced a brief increase of $\sigma_{\phi}$ during 2020. On the other hand, two-thirds of GLONASS satellites were observed with $\sigma_{\phi}>0.1$. These satellites will be discussed in detail in Fig. 2. A third of GLONASS satellites maintained low levels of $\sigma_{\phi}$ for the most part, and these were [R01, R06, R09, R10, R13, R20, R22, and R23]. Through these observations, we conclude that the elevated $\mathrm{L} 1 \sigma_{\phi}$ trends are unique to the GLONASS constellation, as they have not been observed from the other three major GNSS providers during the same period.

Fig. 2 shows the $\sigma_{\phi}$ trends of the 16 most-affected GLONASS satellites. R19 reached the maximum value of

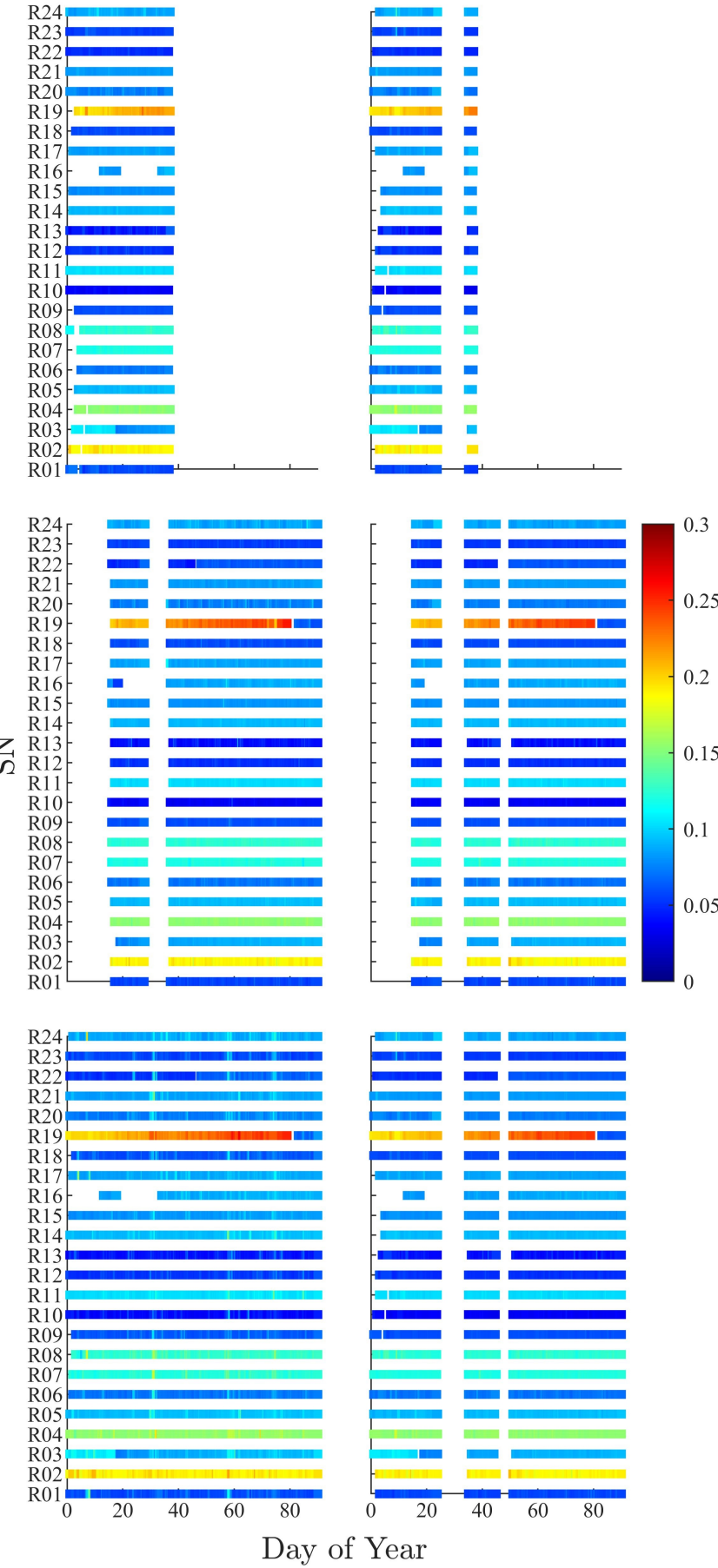

Fig. 3. The 6-hour averaged $\sigma_{\phi}$ observed on GLONASS L1 signal in 2019. The left panel represents the observations from station $\mathrm{m02c}$ (top row) from 01-Jan-2019 to 05-Feb-2019, station $m 12 c$ (middle row) from 16-Jan-2019 to 31-Mar-2019, and station $\mathrm{m07c}$ (bottom row) from 01-Jan-2019 to 31-Mar2019. The right panel represents observations from $S H J 1$ during the same period. The y-axis represents the GLONASS SN, and the $\mathrm{x}$-axis represents the time in day of year. 


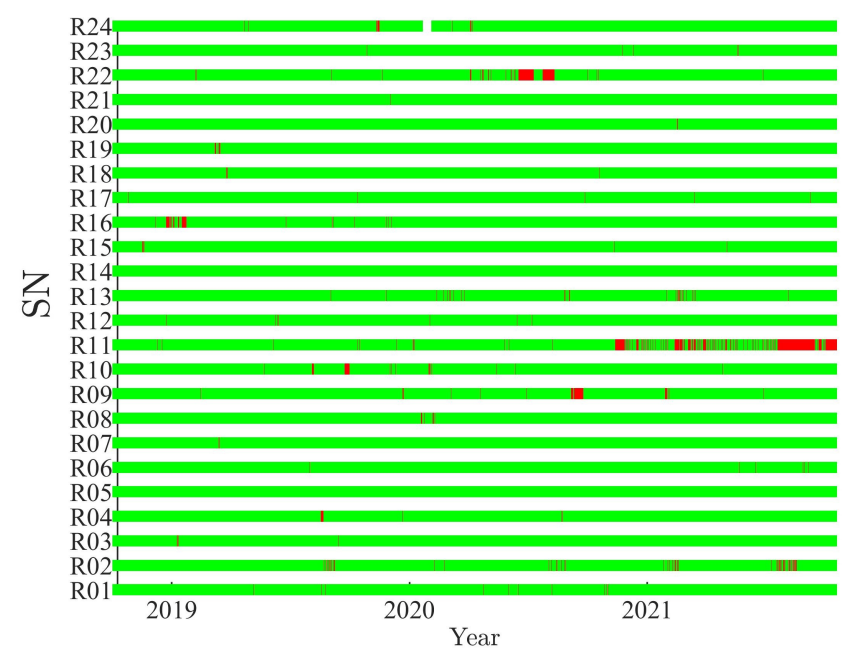

Fig. 4. The health status of GLONASS satellites during three years taken from GLONASS broadcast ephemeris. Green represents satellites flagged as healthy, while red represents satellites that are either unhealthy, unavailable, under maintenance, or testing. The y-axis represents the GLONASS SN, and the $\mathrm{x}$-axis represents the time in years.

$\sigma_{\phi}=0.25$ in the first quarter of 2019. Soon after the observed $\sigma_{\phi}$ was reduced to 0.1 and had been decreasing gradually since. During 2019, R02 approached $\sigma_{\phi}=0.2$; however, its observed $\sigma_{\phi}$ has been decreasing gradually since the second quarter of 2020. A rapid increase of $\sigma_{\phi}$ has been observed on R16 and R24 since 2020 and exceeded 0.15 in 2021. All other GLONASS satellites shown in Fig. 2 have been experiencing a gradual increase in $\sigma_{\phi}$ exceeding $\sigma_{\phi}>0.1$.

In Fig. 3 we compare the 6-hour averaged L1 $\sigma_{\phi}$ observations made at $S H J 1$ and three other stations (see Table. I). These three stations are located in different regions, with $\mathrm{mO2c}$ being at low latitude $\left(18.35^{\circ} \mathrm{N}, 66.75^{\circ} \mathrm{W}\right), \mathrm{m} 12 \mathrm{c}$ being at mid-latitude $\left(51.15^{\circ} \mathrm{N}, 107.26^{\circ} \mathrm{W}\right)$, and $m 07 c$ being at high latitude $\left(64.78^{\circ} \mathrm{N}, 141.16^{\circ} \mathrm{W}\right)$. The main goal of this work is to verify that the observations obtained from $S H J 1$ are in line with observations at different regions.

In Fig. 3, for each row, the set of observations are very similar. The peaking value of $\sigma_{\phi}>0.2$ for R19 can be seen across all stations. Similarly, values of $\sigma_{\phi}>0.1$ can be observed for R02, R04, R07, R08, and R11, across all stations. Therefore, we can deduce that these unnaturally elevated values of $\sigma_{\phi}$ observed from GLONASS satellites from multiple stations are not unique to any specific region, and could not have been caused by any spatial variation, or receiver anomaly.

To ensure that the signals observed were not from satellites that were not fully operational, or those that were not available to simple navigation ground receivers, we present the GLONASS satellite health status in Fig. 4. This data was taken from GLONASS navigation ephemeris files available at the international GNSS service (IGS) broadcast service. The green trends correspond to healthy satellites, whereas the red trends represent satellites that are either unhealthy, unavailable, under maintenance, or testing. Except for R11 in the year 2021, there was no major service outage reported for GLONASS satellites. The service outage of R11 can also be seen in Fig. 1] as observations were no longer available during this period. From Fig. 4, we highlight that the artificially induced phase scintillation observations made in Figs. 13 were from GLONASS satellites labeled as healthy and fit to be used for navigation by the common user.

A commonly used component in GNSS receivers is the Phase Locked Loop (PLL). It aims to minimize the errors between the GNSS signal input phase and the phase output. The presence of phase scintillation on GLONASS signals indicates the presence of rapid phase shifts that may exceed the PLL bandwidth of GNSS receivers. This results in loss of phase lock, which may have serious effects on the reliability and integrity of the GLONASS positioning service [11], [12]. Furthermore, methods such as carrier smoothing that rely on carrier phase measurements to improve positioning precision will also be negatively impacted by these elevated $\sigma_{\phi}$ values [7]. Additionally, abrupt carrier phase discontinuities caused by strong scintillation may degrade the carrier-smoothed pseudo-range accuracy, resulting in considerable positioning errors [13].

\section{CONCLUSION}

In this letter, we have indicated the presence of artificially induced phase scintillation in most GLONASS L1 signals. This feature was not observed on GPS, Galileo, and BeiDou L1 signals during the same period. Observations from different stations at different geographical locations were used to confirm the presence of this anomaly. We have shown that these elevated values of phase scintillation were observed during low solar activity and from satellites labeled as healthy. The reason for conducting this study was to highlight these artificially induced phase scintillations so that future studies may take them into consideration, especially during periods of heightened geomagnetic activity. Furthermore, it is important to note that these artificially induced phase scintillations are detrimental to the reliability and integrity of the GLONASS positioning service by inducing loss of lock to the GNSS user segment, as well as reducing positioning precision. In future work, the effect of the artificially induced GLONASS signals phase scintillation on ground users will be studied, in terms of the lock time as well as the PLL tracking jitter variance.

\section{ACKNOWLEDGMENT}

The authors thank the Monitors for the Alaskan and Canadian Auroral Weather in Space (MACAWS) network for providing GLONASS phase scintillation data, which is freely available http://cedar.openmadrigal.org. The authors also thank the international GNSS service (IGS) for providing GLONASS ephemeris data, which is freely available https://cddis.nasa. gov

\section{REFERENCES}

[1] V. Sreeja, M. Aquino, Z. G. Elmas, and B. Forte, "Correlation analysis between ionospheric scintillation levels and receiver tracking performance," Space Weather, vol. 10, no. 6, 2012. 
[2] T. L. Beach, "Perils of the gps phase scintillation index $(\sigma \phi)$," Radio science, vol. 41, no. 5, 2006.

[3] A. Van Dierendonck, J. Klobuchar, and Q. Hua, "Ionospheric scintillation monitoring using commercial single frequency c/a code receivers," in proceedings of ION GPS, vol. 93. Citeseer, 1993, pp. 1333-1342.

[4] L. Spogli, H. Ghobadi, A. Cicone, L. Alfonsi, C. Cesaroni, N. Linty, V. Romano, and M. Cafaro, "Adaptive phase detrending for gnss scintillation detection: A case study over antarctica," IEEE Geoscience and Remote Sensing Letters, 2021.

[5] P. Prikryl, P. Jayachandran, S. Mushini, and R. Chadwick, "Climatology of gps phase scintillation and hf radar backscatter for the high-latitude ionosphere under solar minimum conditions," in Annales Geophysicae, vol. 29, no. 2. Copernicus $\mathrm{GmbH}, 2011$, pp. 377-392.

[6] P. Prikryl, P. Jayachandran, R. Chadwick, and T. Kelly, "Climatology of gps phase scintillation at northern high latitudes for the period from 2008 to 2013," in Annales Geophysicae, vol. 33, no. $5 . \quad$ Copernicus GmbH, 2015, pp. 531-545.

[7] N. Linty, A. Minetto, F. Dovis, and L. Spogli, "Effects of phase scintillation on the gnss positioning error during the september 2017 storm at svalbard," Space Weather, vol. 16, no. 9, pp. 1317-1329, 2018.

[8] M. Madhanakumar, A. Kashcheyev, and P. Jayachandran, "On the dependence of amplitude and phase scintillation indices on magnetic field aligned angle: A statistical investigation at high latitudes," IEEE Geoscience and Remote Sensing Letters, 2021.

[9] M. M. Shaikh, G. Gopakumar, A. Hussein, A. Kashcheyev, and I. Fernini, "Daytime gnss scintillation due to es over arabian peninsula during low solar activity," Results in Physics, vol. 20, p. 103761, 2021.

[10] Y. Yang, W. Gao, S. Guo, Y. Mao, and Y. Yang, "Introduction to beidou3 navigation satellite system," NAVIGATION, Journal of the Institute of Navigation, vol. 66, no. 1, pp. 7-18, 2019.

[11] M. Aquino and V. Sreeja, "Correlation of scintillation occurrence with interplanetary magnetic field reversals and impact on global navigation satellite system receiver tracking performance," Space Weather, vol. 11, no. 5, pp. 219-224, 2013 .

[12] V. Sreeja, "Impact and mitigation of space weather effects on gnss receiver performance," Geoscience letters, vol. 3, no. 1, pp. 1-13, 2016.

[13] G. T. Myer and Y. T. Morton, "Ionosphere scintillation effects on gps measurements, a new carrier-smoothing technique, and positioning algorithms to improve accuracy," in Proceedings of the 2018 International Technical Meeting of The Institute of Navigation, 2018, pp. 420-439. 